\title{
Fetal Diagnosis and Therapy
}

Abdel-Fattah, S.A. 262

Ackerman, J. 152

Adzick, N.S. 134, 280

Aigrain, Y. 224

Albanese, C.T. 177, 187, 257

Allen, M.H. 152

Anandakumar, C. 212

Apodaca, C.C. 331,338

Aractingi, S. 319

Arduini, D. 8

Ashworth, M. 234

Asnaghi, V. 61

Ayuso, C. 97

Barakonyi, E. 373

Barki, G. 89, 127

Bauerfeind, I. 4

Baxi, L.V. 174

Becker, R. 301

Bekker, M.N. 364

Belics, Z. 373

Bennett, P.R. 102

Ben Said, C. 251

Benzacken, B. 229

Berkane, N. 191, 229

Bernstein, P.S. 165

Bertuzzi, A. 63

Bieganowska, K. 326

Blot, P. 224

Bocchi, C. 8

Boddy, S.-A. 93

Boehm, F.H. 83

Bollmann, R. 79

Bongso, A. 212

Bornick, P.W. 152

Botta, G. 61

Bottoms, S.F. 46

Boulot, P. 132

Bousquet, F. 132

Brackley, K.J. 355

Bruner, J.P. 83, 359

Bucourt, M. 229

Bush, D. 1

Butlin, D. 237

Byrne, D. 234

Byrne, J.L.B. 348

Cabral, A.C.V. 180

Cabrol, D. 41

Cafforio, C. 122

Cagdas, A. 322

Calhoun, B.C. 331,338
Calvano, C.J. 275

Canfield, M.A. 348

Carbillon, L. 251, 308

Carbonne, B. 229

Carles, D. 219

Carreno, C.A. 1

Carroll, S.G. 234, 262

Caruso, A. 246

Cavaliere, A.F. 122,246

Challier, J.-C. 308

Chan, J. 212

Chaoui, R. 79

Chen, S.-M. 108

Chiba, T. 187

Choi, J.M. 174

Cinti, R. 61

Claus, J. 152

Cocheton, J.J. 191

Cohen, E.H. 54

Colosimo, E.A. 180

Cornelis, A. 32

Crescimbini, B. 122

Crombleholme, T.M. 280

Csabay, L. 373

Dadoune, J.P. 229

Dangel, J.H. 326

D'Antona, D. 8

Del Monaco, A. 61

De Santis, M. 246

Deschamps, F. 132

Devlieger, R. 127

Diamant, S. 216

Diamond, M.P. 1

Diard, F. 219

Díaz-Recasens, J. 97

Diniz, J.S.S. 180

Divon, M.Y. 165

Doss, B.J. 342

Dubin, M. 32

Dupont, J.-M. 41

Dupuy, O. 229

Eberhard, J. 283, 291

Entezami, M. 301

Evans, M.I. 1, 46, 89, 331, 335, 338, 342

Evans, W.J. 338

Farmer, D.L. 177, 257, 275

Farrell, J.A. 177, 187, 257

Faure, J.-M. 132
Feldman, B. 89, 335, 342

Feldstein, V.A. 177, 257

Fernández-Moya, J.M. 97

Filly, R.A. 177,187

Fisk, N.M. 102, 118

Flake, A.W. 280

Flore, L.A. 335

Florio, M.G. 122

Floris, M. 170

Forrester, M.B. 146

Franco, J.C. 229

Frey, H. 267

Fujii, T. 304

Fujimoto, S. 112

Ganowicz, J. 326

Gaudet, R. 191

Geissbühler, V. 283, 291

Geka, F. 267

Gembruch, U. 20

Geva, E. 216

Ghysel, C. 127

Gilbert-Barness, E. 152

Girlando, P. 122

Go, A.T.J.I. 364

Goffinet, F. 41

Gole, L.A. 212

Gonzales, M. 229

Goshima, A. 50

Goussot Souchet, M. 41

Graf, J.L. 275

Grangé, G. 41

Gratacós, E. 127

Grisaru-Granovsky, S. 209

Guariglia, L. 63

Guibourdenche, J. 224

Gustafson, O. 71

Hallak, M. 46

Hanatani, K. 112

Harrison, M.R. 177, 187, 257 , 275

Hartung, J. 79

Hasbargen, U. 4

Hattori, R. 112

Hayashi, N. 304

Hepp, H. 4

Hiroi, H. 304

Holder, S.E. 118

Holzgreve, W. 255, 322

Hoshi, N. 112

Hösli, I. 322
Houfflin-Debarge, V. 102

Housley, H.T. 275

Hsieh, F.-J. 108

Hsu, J.-J. 108

Hume, R.F. 331

Hume, R.F., Jr. 338

Ibañez, M.A. 97

Ibba, R.M. 170

Ismail, K.M.K. 355

Iwase, K. 198

Jacques, S.M. 335, 342

Jaffa, A. 216

Jaffe, R. 174

Jarnagin, B.K. 359

Jennings, R.W. 177, 187, 275

Joern, H. 160

Johnson, A. 342

Johnson, M.P. 1, 46, 89, 342

Jou, H.-J. 108

Joye, N. 229

Kajiura, S. 156

Kelly, E.N. 209

Kilby, M.D. 355

King, M. 1

Kirkinen, P. 139

Kishida, T. 112

Kitano, Y. 134

Kjældgaard, A. 71

Kojima, K. 50, 156

Koussef, B. 152

Kozuma, S. 304

Krivchenia, E.L. 89

Kubicka, K. 326

Kyle, P.M. 262

Lagausie, P. de 224

Largillière, C. 251

Lauria, M.R. 46

Leach, R. 89

Le Bourhis, C. 229

Leinonen, M. 139

Leite, H.V. 180

LeRhun, F. 41

Lessing, J.B. 216

Lindton, B. 71

Lipshutz, G.S. 177, 275

Littman, L. 46

Lopoo, J.B. 177 
Lowry, P. 237

Luton, D. 224

McCarthy, L. 93

Machin, G.A. 257

Maeda, K. 198

Maichin, G.A. 177

Maidman, J. 174

Manyonda, I. 237

Maragliano, G. 122

Markling, L. 71

Martin, L.S. 331, 338

Masini, L. 246

Massoni, F. 251

Maugey-Laulom, B. 219

Merviel, P. 191, 308

Merz, R.D. 146

Milliez, J. 229

Minior, V.K. 165

Monni, G. 170

Morales, W.J. 152

Murakami, I. 50, 156

Murakoshi, T. 198

Negishi, H. 112

Német, J. 373

Noia, G. 122, 246

O’Donnell, H. 102

Ohki, S. 198

Okai, T. 304

Okajima, K. 50

Okuyama, K. 112

Oliva, N. 246

Oliveira, E.A. 180

Ordén, M.-R. 139

Oury, J.F. 224

Overton, T.G. 102, 118

Oya, N. 156

Paek, B.W. 4, 177

Page, N. 237
Pannier, E. 41

Papp, Z. 373

Pereira, A.K. 180

Peuchmaur, M. 224

Pierce, B. 331

Portnoi, M.-F. 229

Pryde, P.G. 46

Pujazon, M.A. 348

Putzolu, M. 170

Quintero, R.A. 152

Qureshi, F. 335, 342

Ramos, C. 97

Rath, W. 160

Rein, A.J.J.T. 54

Reinisch, L. 359

Richards, W.O. 83

Ringdén, O. 71

Rizzo, G. 8

Robledo, M. 97

Rodríguez de Alba, M. 97

Rosati, P. 63

Roszkowski, T. 326

Ruchelli, E. 134

Runkel, S. 301

Ryan, G. 209

Sagawa, T. 112

Salvego, M. 61

Samuel, M. 93

Sandberg, P.L. 177, 257

Sanz, R. 97

Scapillati, M.E. 122

Scharf, A. 267

Scheuerle, A.E. 348

Schlemmer, A. 267

Schouppe, S. 191

Seaward, P.G.R. 209

Segondy, M. 132

Seguchi, M. 198
Senat, M.V. 36

Serizawa, M. 198

Seubert, D.E. 89

Severi, F.M. 8

Sewry, C.A. 118

Shi, C.-y. 20

Shih, J.-C. 108

Shikada, T. 50

Shyu, M.-K. 108

Sibony, O. 224

Siffroi, J.P. 229

Silengo, M. 61

Smith, R.P. 118

Smrcek, J.M. 20

Sohan, K. 234

Sohn, C. 267

Soothill, P.W. 234, 262

Soussotte, C. 219

Steinborn, A. 267

Strauss, A. 4

Strauss, S. 216

Studer, C. 229

Suzuki, Y. 50, 156

Suzumori, K. 50, 156

Sylvester, K. 280

Szabó, I. 373

Taillemite, J.-L. 229

Takagi, T. 112

Taketani, Y. 304

Tanemura, M. 156

Tercanli, S. 322

Terry, M. 331

Thomas, D.G. 335

Thoury, A. 41

Tortorolo, G. 122

Tsutsumi, O. 304

Tulipan, N.B. 83

Unno, N. 304

Utsu, M. 198

Uyanik, G. 322
Uzan, M. 229, 251, 308

Uzan, S. 191, 229, 308, 319

Valk, J. 364

Van Buchem, M.A. 364

Van den Bosch, T. 32

Vandenbussche, F.P.H.A. 364

Van Schoubroeck, D. 32

Van Vugt, J.M.G. 364

Verzuri, M.S. 8

Vilasboas, A.S. 180

Ville, Y. 36

Vrabcak, E.K. 83

Wagenvoort, A.M. 364

Walker, W. 331

Waller, D.K. 348

Walsh, W.F. 83

Weiner, S. 134

Westgren, M. 71

Windrim, R. 209

Wolf, J.P. 229

Wolman, I. 216

Wong, Y.C. 212

Wright, J.G.C. 355

$\mathrm{Wu}$, J. 127

Wyatt, P. 209

Yamada, H. 112

Yamamoto, N. 198

Yamamoto, T. 50

Yamashita, N. 156

Yang, R. 212

Yaron, Y. 1, 216

Yesildaglar, N. 127

Zuppa, A.A. 122 